\title{
Accelerating the development of therapeutic strategy for oligometastasis
}

\author{
Yoshihisa Shimada \\ Department of Thoracic Surgery, Tokyo Medical University, Japan, Tokyo \\ Correspondence to: Yoshihisa Shimada, MD, PhD. Department of Thoracic Surgery, Tokyo Medical University, 6-7-1, Nishi Shinjuku, Shinjuku-ku, \\ Tokyo 160-0023, Japan. Email: zenkyu@za3.so-net.ne.jp. \\ Provenance: This is an invited article commissioned by the Section Editor Xiaozheng Kang (Department of Thoracic Surgery, Beijing Cancer \\ Hospital, Peking University, Beijing, China). \\ Comment on: Dingemans AC, Hendriks LEL, Berghmans T, et al. Definition of synchronous oligo-metastatic non-small cell lung cancer - a consensus \\ report. J Thorac Oncol 2019;14:2109-19.
}

Submitted Nov 17, 2019. Accepted for publication Dec 09, 2019.

doi: $10.21037 /$ jtd.2019.12.42

View this article at: http://dx.doi.org/10.21037/jtd.2019.12.42

Metastasis is the principal cause of death among a large majority of cancer patients because of their systemic nature and the resistance of disseminated tumor cells to existing therapeutic agents. Patients who developed distant metastasis from lung cancers had been best served with palliative-intent systemic therapies. However, recent advances in lung cancer treatment including immune checkpoint inhibitors, molecular-target agents, and less invasive local therapies such as minimally-invasive surgery or stereotactic ablative radiotherapy (SAbR) have accelerated the introduction of a "game-changing" curative intent approach in patients with limited metastatic burden, termed "oligometastasis". There has been an increasing interest in the use of local ablative therapy (LAT) for patients with metastatic disease with the purpose of longterm survival or even cure.

As a sequel to the spectrum theory of cancer spread, Hellman and Weichselbaum proposed the existence of the oligometastatic state in 1995 (1). They suggested that oligometastasis is a limited number of metastases that exists at first before cancer cells acquire widespread metastatic potential, representing an intermediate state as well as the transient phase between the early localized disease and diffuse metastasis. To form metastasis, cancer cells undergo a multi-step biological process from local invasion to the metastatic formation (2). This metastatic cascade suggests that oligometastasis is different from polymetastasis on different steps of metastases development (3). From a clinical point of view, patients with oligometastatic state is associated with indolent tumor behavior, manifested by a long-term survival, a limited number of metastases, and favorable tumor histology (4-6). Therefore, oligometastatic state does not appear to progress to the widespread distribution of cancer.

Prospective randomized trials have demonstrated promising favorable long-term outcomes for patients with oligometastasis with the use of LAT in comparison with standard-of-care systemic therapy alone (7-13). For nonsmall cell lung cancer (NSCLC), two recent randomized phase II studies demonstrated improved progression-free survival (PFS) in patients with oligometastatic NSCLC who were treated with LAT compared with systemic therapy alone (7-9). In the Gomez study, 49 patients with stage IV NSCLC with 3 of fewer metastases, and no disease progression after front-line systemic therapy, were assigned to receive either LAT to all active disease sites or maintenance systemic therapy alone $(7,8)$. There were significant differences shown in PFS of 4 vs. 12 months as well as OS of 41 vs. 17 months $(7,8)$. The second study was a single-institutional phase 2 study to compare maintenance chemotherapy alone with SAbR followed by maintenance chemotherapy for 29 patients with up to 5 metastases, and the study was stopped early due to an interim analysis that revealed a significantly improved PFS in the local control group (9.7 vs. 3.5 months) (9). Besides, preceded several single-arm clinical studies in oligometastatic NSCLC 
proved that LAT contributed to favorable long-term survival (14-18). Oligometastatic NSCLC has increasingly been recognized as a unique clinical state with therapeutic implications, described as a unique therapeutic entity in the European Society of Medical Oncology guidelines, the National Comprehensive Cancer Network guideline, and the $8^{\text {th }}$ tumor-node-metastasis classification (19-22). However, there is no universal definition, with the heterogeneity of clinically defined oligometastatic lung cancer in terms of the number or location of clinically detectable metastases, the interval between treating the primary tumor and detection of metastasis, and the volume of disease. Given that LAT can be responsible for a risk of healthy tissue damage or impaired quality of life, and skepticism remains over the efficacy of LAT for metastatic disease because of the likelihood of occult metastases, a more homogeneous and unique definition of oligometastatic NSCLC is warranted.

A consensus report that was recently published in the Fournal of Thoracic Oncology on the definition and staging of oligometastatic NSCLC is expected to be highly useful and important for summarizing current controversies and definitions as preparatory works for patient selection in future clinical trials (23). Thirty-five thoracic oncology experts from different societies and European countries compiled a consensus definition for synchronous oligometastatic NSCLC. This consensus process consists of 3 preparatory works; (I) a survey, (II) real-life cases, and (III) a systematic review (23).

A survey was sent to the consensus group, national societies, and members of the EORTC lung cancer and radiation oncology groups, and 423 physicians from 34 countries completed the survey. The systematic review identified 21 papers that were eligible for the systematic analysis and included 1,215 patients with oligometastatic NSCLC (24). Although there was variation from 1 to 8 metastatic sites allowed in the definition of oligometastatic NSCLC, less than 5 metastases were allowed in the majority of papers. For the 10 real cases of oligometastatic NSCLC, there was a $100 \%$ consensus in 1 case, over $90 \%$ consensus in 3 cases, and $38 \%$ to $68 \%$ for the rest on the diagnosis of oligometastatic disease. These results were summarized at the consensus meeting. In brief, the consensus findings of oligometastatic NSCLC provided the aim of treatment, definition, and staging of oligometastatic NSCLC. According to the review, a maximum of 5 metastases and 3 organs were agreed on. The authors stated that there were a wide variety of opinions and no consensus was obtained on this point. The survey showed that a maximum of 3 metastatic sites was the most frequent responses while the systemic review indicated that up to 5 metastases was the most frequent definition. It is likely that most patients who had oligometastatic disease actually harbor occult metastases at the time of treatment. Unless better predictive and prognostic biomarkers can be identified to determine which patients harbor occult metastases that will progress, the maximum number of metastases and organs by a certain threshold should be defined to overcome barriers to accrual for randomized clinical trials. In the end, the authors concluded that more than 5 metastases should not be included in the definition since oligo represents "few" and with support from published data.

Another interesting propose of this definition is the nature of the organs involved. The authors proclaimed that all organs are subjected to be treated, except diffuse serosal metastases. Brain, adrenal and pulmonary metastases were considered suitable sites for LAT because there are many retrospective data on oligometastasis in these organs. However, with the development of advanced local treatment techniques of radiation therapies or surgery, recent advances in immune checkpoint inhibitors or targeted-therapy are likely to give new LAT roles in terms of safety and efficacy by the integrated therapies even in patients with oligometastases in other metastatic sites such as liver, bone, or skin.

The staging of oligometastatic NSCLC including imaging workup by means of 18F-FDG-PET-CT as well as MRI for the detection of brain metastasis was also a very important issue. Imaging requirements for recognizing oligometastatic NSCLC vary with the location of metastatic and measurement timing regarding to previous treatment. At each point in the disease course, imaging must be tailored to the context of prior treatment. Optimal standardized imaging approaches will allow us to document disease progression patterns and treatment outcomes on future prospective trials.

This consensus work is an important milestone that can help us speak the same language about oligometastatic NSCLC. However, we are just standing at the starting point for the strategy of treating oligometastatic NSCLC with LAT in order to gain widespread availability as routine practice, and there are still many issues to be addressed. For example, which biological effective dose of radiation therapy would be recommended to produce convincing efficacy to different metastatic states and metastatic locations in actual practice? As aggressive systemic 
treatment followed by surgery should be very toxic, which population of oligometastatic NSCLC can tolerate both? Ultimately the arrival of future prospective randomized phase III trial data with enrolled NSCLC patients bases on this multidisciplinary consensus statement and a better understanding of biology will hopefully lead to stronger evidence of LAT supporting its real efficacy.

\section{Acknowledgments}

None.

\section{Footnotes}

Conflicts of Interest: The author has no conflicts of interest to declare.

Ethical Statement: The author is accountable for all aspects of the work in ensuring that questions related to the accuracy or integrity of any part of the work are appropriately investigated and resolved

\section{References}

1. Hellman S, Weichselbaum RR. Oligometastases. J Clin Oncol 1995;13:8-10.

2. Valastyan $S$, Weinberg RA. Tumor metastasis: molecular insights and evolving paradigms. Cell 2011;147:275-92.

3. Hellman S. Karnofsky Memorial Lecture. Natural history of small breast cancers. J Clin Oncol 1994;12:2229-34.

4. Ashworth AB, Senan S, Palma DA, et al. An individual patient data metaanalysis of outcomes and prognostic factors after treatment of oligometastatic non-small-cell lung cancer. Clin Lung Cancer 2014;15:346-55.

5. Griffioen GH, Toguri D, Dahele M, et al. Radical treatment of synchronous oligometastatic non-small cell lung carcinoma (NSCLC): patient outcomes and prognostic factors. Lung Cancer 2013;82:95-102.

6. Tanvetyanon T, Robinson LA, Schell MJ, et al. Outcomes of adrenalectomy for isolated synchronous versus metachronous adrenal metastases in non-small-cell lung cancer: a systematic review and pooled analysis. J Clin Oncol 2008;26:1142-7.

7. Gomez DR, Blumenschein GR Jr, Lee JJ, et al. Local consolidative therapy versus maintenance therapy or observation for patients with oligometastatic non-smallcell lung cancer without progression after first-line systemic therapy: a multicentre, randomised, controlled, phase 2 study. Lancet Oncol 2016;17:1672-82.

8. Gomez DR, Tang C, Zhang J, et al. Local Consolidative Therapy Vs. Maintenance Therapy or Observation for Patients With Oligometastatic Non-Small-Cell Lung Cancer: Long-Term Results of a Multi-Institutional, Phase II, Randomized Study. J Clin Oncol 2019;37:1558-65.

9. Iyengar P, Wardak Z, Gerber DE, et al. Consolidative Radiotherapy for Limited Metastatic Non-Small-Cell Lung Cancer: A Phase 2 Randomized Clinical Trial. JAMA Oncol 2018;4:e173501.

10. Ost P, Reynders D, Decaestecker K, et al. Surveillance or Metastasis-Directed Therapy for Oligometastatic Prostate Cancer Recurrence: A Prospective, Randomized, Multicenter Phase II Trial. J Clin Oncol 2018;36:446-53.

11. Palma DA, Olson R, Harrow S, et al. Stereotactic ablative radiotherapy versus standard of care palliative treatment in patients with oligometastatic cancers (SABRCOMET): a randomised, phase 2, open-label trial. Lancet 2019;393:2051-8.

12. Ruers T, Punt C, Van Coevorden F, et al. Radiofrequency ablation combined with systemic treatment versus systemic treatment alone in patients with non-resectable colorectal liver metastases: a randomized EORTC Intergroup phase II study (EORTC 40004). Ann Oncol 2012;23:2619-26.

13. Ruers T, Van Coevorden F, Punt CJ, et al. Local Treatment of Unresectable Colorectal Liver Metastases: Results of a Randomized Phase II Trial. J Natl Cancer Inst 2017;109. doi: 10.1093/jnci/djx015.

14. Collaud S, Stahel R, Inci I, et al. Survival of patients treated surgically for synchronous single-organ metastatic NSCLC and advanced pathologic TN stage. Lung Cancer 2012;78:234-8.

15. Congedo MT, Cesario A, Lococo F, et al. Surgery for oligometastatic non-small cell lung cancer: longterm results from a single center experience. J Thorac Cardiovasc Surg 2012;144:444-52.

16. De Ruysscher D, Wanders R, van Baardwijk A, et al. Radical treatment of non-small-cell lung cancer patients with synchronous oligometastases: long-term results of a prospective phase II trial (Nct01282450). J Thorac Oncol 2012;7:1547-55.

17. Johnson KK, Rosen JE, Salazar MC, et al. Outcomes of a Highly Selective Surgical Approach to Oligometastatic Lung Cancer. Ann Thorac Surg 2016;102:1166-71.

18. Inoue $\mathrm{T}$, Katoh $\mathrm{N}$, Aoyama H, et al. Clinical outcomes of stereotactic brain and/or body radiotherapy for patients with oligometastatic lesions. Jpn J Clin Oncol 2010;40:788-94. 
19. Eberhardt WE, Mitchell A, Crowley J, et al. The IASLC Lung Cancer Staging Project: Proposals for the Revision of the M Descriptors in the Forthcoming Eighth Edition of the TNM Classification of Lung Cancer. J Thorac Oncol 2015;10:1515-22.

20. Ettinger DS, Aisner DL, Wood DE, et al. NCCN Guidelines Insights: Non-Small Cell Lung Cancer, Version 5.2018. J Natl Compr Canc Netw 2018;16:807-21.

21. Novello S, Barlesi F, Califano R, et al. Metastatic nonsmall-cell lung cancer: ESMO Clinical Practice Guidelines for diagnosis, treatment and follow-up. Ann Oncol 2016;27:v1-v27.

Cite this article as: Shimada Y. Accelerating the development of therapeutic strategy for oligometastasis. J Thorac Dis 2019;11(12):5670-5673. doi: 10.21037/jtd.2019.12.42
22. Planchard D, Popat S, Kerr K, et al. Metastatic non-small cell lung cancer: ESMO Clinical Practice Guidelines for diagnosis, treatment and follow-up. Ann Oncol 2018;29:iv192-iv237.

23. Dingemans AC, Hendriks LEL, Berghmans T, et al. Definition of Synchronous Oligometastatic Non-Small Cell Lung Cancer-A Consensus Report. J Thorac Oncol 2019;14:2109-19.

24. Giaj-Levra N, Giaj-Levra M, Durieux V, et al. Defining Synchronous Oligometastatic Non-Small Cell Lung Cancer: A Systematic Review. J Thorac Oncol 2019;14:2053-61. 